\title{
Comparative study of DIPSI and IADPSG criteria for diagnosis of GDM
}

\author{
Swathy Srinivasan*, Rani P. Reddi
}

Department of Obstetrics and Gynecology, Mahatma Gandhi Medical College and Research Institute, Puducherry, India

Received: 14 December 2017

Accepted: 20 January 2018

*Correspondence:

Dr. Swathy Srinivasan,

E-mail: drswathyyy@gmail.com

Copyright: () the author(s), publisher and licensee Medip Academy. This is an open-access article distributed under the terms of the Creative Commons Attribution Non-Commercial License, which permits unrestricted non-commercial use, distribution, and reproduction in any medium, provided the original work is properly cited.

\begin{abstract}
Background: GDM is defined as any degree of glucose intolerance with onset or first recognition during pregnancy. The prevalence of GDM varies, widely based on the diagnostic criteria used and the ethnic group studied. It is associated with adverse maternal and perinatal outcome. Incidence of GDM in India is 1-14\%. There are several screening and diagnostic tests for GDM. It is important to diagnose early and treat to prevent these complications. The present study was done to compare Diabetes in Pregnancy Study Group India (DIPSI) with International Association of the Diabetes and Pregnancy Study Groups (IADPSG) criteria for diagnosis of GDM and to assess the validity of these methods.

Methods: It was a cross sectional study done in 144 pregnant women who fulfilled the inclusion criteria. They underwent non - fasting OGTT with 75 grams glucose which was given irrespective of the last meal. A venous blood sample was drawn two hours after glucose administration. They were advised to come two to three days later and repeated with 75 grams OGTT after an overnight fast of atleast 8 hours. Venous blood sample was drawn at fasting, one hour and two hours after load with 75 grams of glucose. Plasma glucose was measured by using an autoanalyzer by glucose - oxidase peroxidase (GOD - POD) technique.

Results: The epidemiological parameters like Age, BMI, Parity and Gestational age did not have any difference between two groups. $17.4 \%$ was diagnosed by DIPSI criteria and $15.3 \%$ was diagnosed by IADPSG criteria and $6.9 \%$ was diagnosed by both. Sensitivity and specificity of DIPSI was $45 \%$ and87\% and sensitivity and specificity of IADPSG was $40 \%$ and $89 \%$ respectively. According to kappa statistics, the p-value is 0.000 .

Conclusions: In present study it was concluded that screening is very essential in all pregnant women due to high prevalence of GDM in India. By comparing these two criteria, sensitivity of DIPSI was found better than IADPSG criteria in diagnosing GDM. Though IADPSG is universally accepted for diagnosis, DIPSI has still got a place in low resource countries as it is easy, cost effective and non fasting test.
\end{abstract}

Keywords: DIPSI, GDM, IADPSG

\section{INTRODUCTION}

GDM is defined as any degree of glucose intolerance with onset or first recognition during pregnancy. ${ }^{1}$ The prevalence of GDM varies, widely based on the diagnostic criteria used and the ethnic group studied. ${ }^{2}$ It is associated with adverse maternal and perinatal outcome.
Therefore, screening is essential in all pregnant women in Asia as they are at 11 fold increased risk of developing glucose intolerance during pregnancy as compared to caucasian women. ${ }^{3}$

GDM has been found to be more common in women living in urban areas as compared to women living in 
rural areas. ${ }^{4}$ So it is important to diagnose early and treat to prevent the morbidity. There are different types of screening methods viz. universal or risk based, one step or two step and different thresholds for diagnosis.

In India most centres do one step screening and diagnostic method with Diabetes in Pregnancy Study Group India (DIPSI) as it is simple, easy, feasible in non fasting state but it has low sensitivity and diurnal variation causing controversy. ${ }^{5,6}$ It has been observed by Hyperglycemia and Pregnancy Outcome (HAPO) study that The International Association of the Diabetes and Pregnancy Study Groups (IADPSG) criteria has more sensitivity, universally accepted and even an isolated fasting glucose levels have higher incidence of poor maternal and fetal outcome. The present study was done to compare DIPSI (non-fasting OGTT) with IADPSG criteria for diagnosis of GDM and to assess the validity of these methods.

\section{METHODS}

This is a cross sectional study of 144 pregnant women who were fulfilling the inclusion criteria and willing to participate in the study were included. Informed and written consent were taken before study and ethical committee approval was obtained. A standardized questionnaire was used to collect demographic details, family history of diabetes, previous history of Gestational Diabetes Mellitus, previous obstetric history. Height was measured by stadiometer in centimeter, weight by electronic weighting machine in kilograms. The BMI was calculated by weight (in $\mathrm{kg}$ ) divided by height in meter (squared) and any other associated risk factors was also noted. They underwent non - fasting OGTT with 75 grams glucose which was given irrespective of the last meal. A venous blood sample was drawn two hours after glucose administration. They were advised to come two to three days later and repeated with 75 grams OGTT after an overnight fast of atleast 8 hours. Venous blood sample was drawn at fasting, one hour and two hours after load with 75 grams of glucose. Plasma glucose was measured by using an autoanalyzer by glucose - oxidase peroxidase (GOD - POD) technique.

\section{Inclusion criteria}

All pregnant women attending antenatal OPD of Mahatma Gandhi Medical College and Research Institute over a period of 18 months with the gestation of 20-32 weeks.

\section{Exclusion criteria}

- Pregnant women with known pregestational diabetes mellitus

- Pregnant women with chronic illness (renal, pancreatic etc.)

- Pregnant women on drugs like corticosteroids, hydrochlorothiazide and antipsychotic drugs.

\section{Statistical analysis}

All data was entered into Microsoft excel 2007. It was analysed by using SPSS Windows (Version 16). Descriptive data was expressed by using number and simple percentage. Sensitivity and Specificity was calculated and compared between DIPSI and IADPSG criteria.

\section{RESULTS}

The epidemiological parameters like Age, BMI, Parity, Gestational age did not have any difference between two groups. $68 \%$ of women were primigravida and $31 \%$ of women were multigravida among 144 patients screened.

Table 1: Comparison of mean age between positive cases of DIPSI and IADPSG criteria.

\begin{tabular}{|lllllll|}
\hline Age & Positive & Mean & Median & Standard deviation & t-test & p-value \\
\hline DIPSI & 25 & 25.96 & 26 & 4.04 & 1.2826 & 0.2062 \\
\cline { 1 - 2 } & 22 & 27.36 & 27.5 & 3.35 & & \\
\hline
\end{tabular}

Among the 25 positive women in DIPSI the mean age was 25.96, median was 26 and standard deviation was 4 and among the 22 IADPSG positive women the mean age was 27.36 , median was 27 and standard deviation was 3 .

$\mathrm{P}$-value was not significant between the two groups.

Table 2: Comparison of BMI between positive cases of DIPSI and IADPSG criteria.

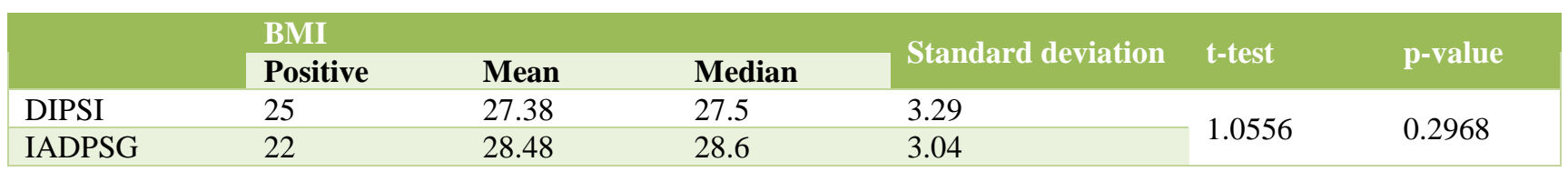


By comparing the BMI among the 25 DIPSI positive the mean was 27.38 , median was 27.5 and standard deviation was 3.

In IADPSG criteria among the 22 positive the mean was 28.48, median was 28 and standard deviation was 3. Pvalue was not significant between the two groups.

Table 3: Distribution of patients according to gestational age in all screened patients.

\begin{tabular}{|lll|}
\hline Gestational age (weeks) & No. of cases & Percentage \\
\hline $20-25$ & 80 & 55.6 \\
\hline $26-32$ & 64 & 44.4 \\
\hline Total & 144 & 100 \\
\hline
\end{tabular}

About $56 \%$ of women were within 20-25 weeks of gestational age and $44 \%$ of women were within $26-32$ weeks of gestational age.
Table 4: Total number of positive and negative cases by DIPSI criteria.

\begin{tabular}{|lll|}
\hline DIPSI & No. of cases & Percentage \\
\hline Positive & 25 & 17.4 \\
\hline Negative & 119 & 82.6 \\
\hline Total & 144 & 100 \\
\hline
\end{tabular}

Total number of positive cases by DIPSI was 25 and their percentage is $17.4 \%$ and total number negative cases of DIPSI was 119 and their percentage is $82 \%$.

Table 5: Total number of positive and negative cases by IADPSG criteria.

\begin{tabular}{|lll|}
\hline IADPSG & No. of cases & Percentage \\
\hline Positive & 22 & 15.3 \\
\hline Negative & 122 & 84.7 \\
\hline Total & 144 & 100 \\
\hline
\end{tabular}

Table 6: Statistical parameters between positive and negative cases of DIPSI and IADPSG criteria.

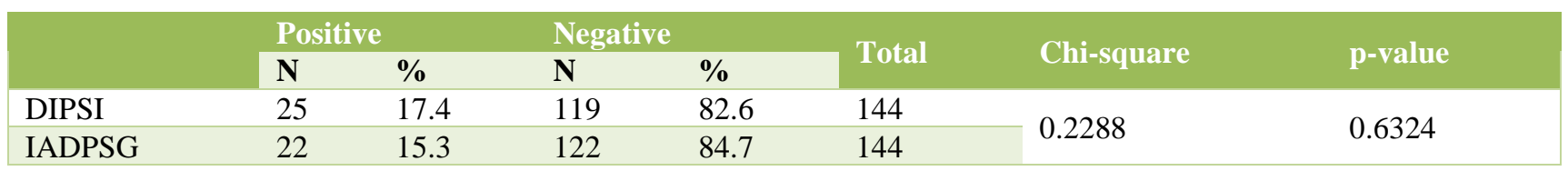

Total number of positive cases of IADPSG was 22 and their percentage is $15.3 \%$ and total number of negative cases of IADPSG was 122 and their percentage is $84.7 \%$.

In parameters between both positive and negative cases of DIPSI and IADPSG criteria the chi-square was 0.2288 and p-value was 0.6 and was not statistically significant.

Table 7: Comparison of DIPSI with IADPSG criteria.

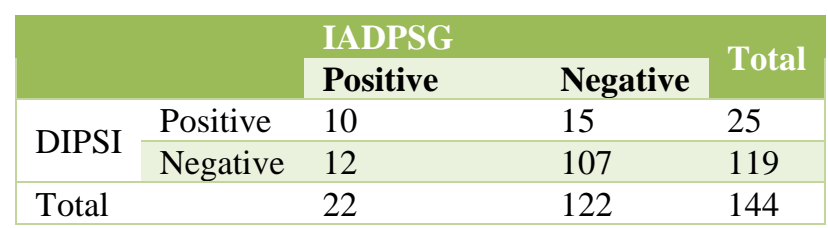

Number of both DIPSI and IADPSG positive in 10 cases, both negative in 107, DIPSI positive with IADPSG negative has 15 , DIPSI negative with IADPSG positive has 12 .

The sensitivity of DIPSI with IADPSG was $45.45 \%$, specificity $87.70 \%$, Positive predictive value $40.00 \%$ and Negative predictive value $89.92 \%$.

Number of both IADPSG and DIPSI positive was 10 cases, both negative were 107, IADPSG positive with
DIPSI negative was 12, IADPSG negative with DIPSI positive was 15 cases.

Table 8: Statistical parameters of DIPSI with IADPSG criteria.

\begin{tabular}{|lll|}
\hline Statistical parameters & Value & $95 \%$ CI \\
\hline Sensitivity & $45.45 \%$ & $24.39 \%$ to $67.79 \%$ \\
\hline $\begin{array}{l}\text { Specificity } \\
\text { Positive likelihood }\end{array}$ & $87.70 \%$ & $80.53 \%$ to $92.95 \%$ \\
\hline $\begin{array}{l}\text { ratio } \\
\text { Negative likelihood } \\
\text { ratio }\end{array}$ & 0.62 & 0.42 to 0.92 \\
\hline $\begin{array}{l}\text { Disease prevalence } \\
\text { Positive predictive } \\
\text { value }\end{array}$ & $15.28 \%$ & $9.83 \%$ to $22.21 \%$ \\
\hline $\begin{array}{l}\text { Negative predictive } \\
\text { value }\end{array}$ & $80.00 \%$ & $25.65 \%$ to $56.30 \%$ \\
\hline
\end{tabular}

Table 9: Comparison between IADPSG and DIPSI criteria.

\begin{tabular}{|c|c|c|c|c|}
\hline & \multicolumn{2}{|l|}{ DIPSI } & \multirow{2}{*}{ Total } \\
\hline & & Positive & Negative & \\
\hline \multirow{2}{*}{ IADPSG } & Positive & 10 & 12 & 22 \\
\hline & Negative & 15 & 107 & 122 \\
\hline Total & & 25 & 119 & 144 \\
\hline
\end{tabular}


According to kappa statistic (Kappa value 0.314, P-value $0.000)$, there is disagreement in diagnosing GDM between DIPSI and IADPSG criteria.

Table 10: Statistical parameters between IADPSG with DIPSI criteria.

\begin{tabular}{|lll|}
\hline $\begin{array}{l}\text { Statistical } \\
\text { parameters }\end{array}$ & Value & $95 \%$ CI \\
\hline $\begin{array}{l}\text { Sensitivity } \\
\text { Specificity }\end{array}$ & $80.00 \%$ & 21.13 to $61.33 \%$ \\
\hline $\begin{array}{l}\text { Positive likelihood } \\
\text { Ratio }\end{array}$ & 3.97 & 1.93 to 8.15 \\
\hline $\begin{array}{l}\text { Negative likelihood } \\
\text { ratio }\end{array}$ & 0.67 & 0.48 to 0.92 \\
\hline $\begin{array}{l}\text { Disease prevalence } \\
\text { Positive predictive } \\
\text { value }\end{array}$ & $17.36 \%$ & $11.56 \%$ to $24.55 \%$ \\
\hline $\begin{array}{l}\text { Negative predictive } \\
\text { value }\end{array}$ & $87.45 \%$ & $28.86 \%$ to $63.13 \%$ \\
\hline
\end{tabular}

The sensitivity of IADPSG is $40.00 \%$, specificity is $89.92 \%$, positive predictive value is $45.45 \%$ and negative predictive value is $87.70 \%$ when compared to DIPSI. Therefore, by comparing tables 17 and 19 DIPSI has got higher sensitivity and is found comparatively better than IADPSG in diagnosing GDM.

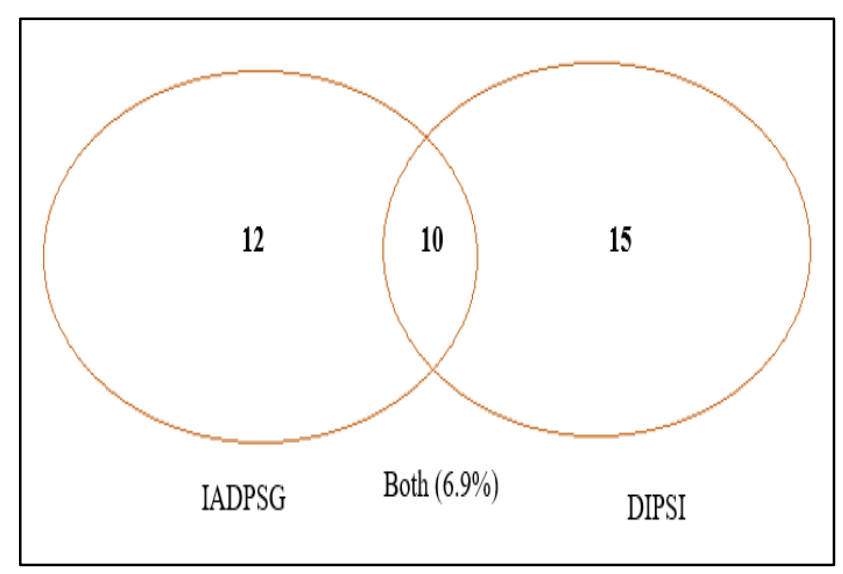

Figure 1: Total number of DIPSI and IADPSG positive.

Both were positive in 10 cases. DIPSI diagnosed higher number of GDM cases than IADPSG.

\section{DISCUSSION}

This was a cross sectional study carried out over a period of 18 months from March 2016 to August 2017 in antenatal women who were attending MGMCRI OG OPD, who were within the gestational age of 20 to 32 weeks, who fulfilled the inclusion criteria and were willing to participate in the study.

Gestational diabetes mellitus remains as one of the major public health problem associated with adverse maternal and perinatal outcome. The prevalence is completely based on the diagnostic criteria and the ethnic group studied. There is a lack of uniformity in screening and diagnostic tests for GDM and there is a need for universal screening for GDM in high risk population like Indians. A simple, easier, cost effective, non fasting test DIPSI is being followed widely in India.

Recently based on HAPO study IADPSG consensus panel recommended that GDM should be diagnosed based on IADPSG criteria which has more sensitivity and specificity, more precise and accurate for diagnosing GDM and to have uniform diagnosing method all over the world. India was not included in HAPO study inspite of being highly populous and high risk ethnic group. The present study was conducted with the aim of comparing the sensitivity and specificity of DIPSI and IADPSG criteria for diagnosis of GDM and to evaluate the maternal and perinatal complications.

In the present study, the epidemiological parameters like age, BMI, parity, family history of Diabetes Mellitus and Gestational age at screening did not have any difference in both groups and not statistically significant. $68 \%$ were primigravida and $40 \%$ had family history of Diabetes Mellitus. Similar findings were observed in studies by Mohan et al, Geetha et al and Pulkit et al., 5,7,8

In the present study, out of 25 women identified as GDM by DIPSI criteria, only 10 women were diagnosed by IADPSG. Of the 22 women diagnosed by IADPSG, 12 women were not detected by DIPSI criteria. The prevalence of GDM in the present study was $17.4 \%$ (25) with DIPSI and $15.3 \%$ (22) based on IADPSG criteria. Both tests were positive in only $6.9 \%$ (10). The sensitivity of DIPSI was $45.45 \%$, specificity was $87.70 \%$, positive predictive value was $40.00 \%$ and negative predictive value was $89.92 \%$.

The sensitivity of IADPSG was $40.00 \%$, specificity was $89.92 \%$, positive predictive value was $45.45 \%$ and negative predictive value was $87.70 \%$. In the present study the sensitivity of DIPSI was slightly higher and was found to be comparatively better than IADPSG criteria for diagnosis of GDM. According to Kappa statistic the p-value was 0.000 .

Geetha et al in their prospective study of 100 women found prevalence rates of GDM were $14 \%$ and $9 \%$ with DIPSI and IADPSG criteria respectively and $4 \%$ were diagnosed by both leaving 5\% undiagnosed by IADPSG criteria which could have been easily detected by DIPSI. ${ }^{7}$ Though DIPSI criteria detected more number of cases than IADPSG criteria the difference was not statistically significant. They concluded that DIPSI was better than IADPSG criteria as it was easier to do and tolerance of patient to glucose load was much better in non fasting state whereas IADPSG needed more blood samplings, fasting state, more laboratory support with extra cost. In low resource settings, DIPSI is better than IADPSG for 
diagnosis of GDM. The observations of this study were similar to present study. In a mini review on diagnostic criteria for GDM the authors emphasized DIPSI as cost effective and evidence based procedure in the low resource countries and with high risk ethnic population like Indians who require universal screening as a single step definitive glucose test. ${ }^{9}$

Pulkit et al in their retrospective study of 152 women comparing DIPSI and IADPSG criteria and also evaluating the isolated fasting glucose in diagnosis of GDM found that IADPSG criteria found better than DIPSI as it left $23.36 \%$ undiagnosed which were easily detected by IADPSG criteria and they found prevalence rate of GDM was $74.34 \%$ by DIPSI and $88.15 \%$ by IADPSG using both fasting and 2 hours plasma glucose values and by fasting value of $\geq 92 \mathrm{mg} / \mathrm{dl} 22.365 \%$ were diagnosed. ${ }^{8}$ They claimed that higher rates were due to prevalence of high risk factors in their subjects of study like advanced age, BMI, family history etc and also associated with poor maternal and fetal outcome and justified that IADPSG criteria was better as it picked up more number of women with GDM than DIPSI and also by analyzing the complications associated with GDM patients IADPSG criteria serves better than DIPSI.

Bhavadharini et al highlighted the GDM diagnosis strategy based on women in India with GDM Strategy (WINGS) project carried out in Chennai suggested that despite the constraints of low resource, fasting state, three blood samples in IADPSG criteria, it appears to be the best which will help to bring out a uniform criteria for screening and diagnosis of GDM. ${ }^{10}$

DIPSI criteria which is used widely in many parts of India due to its simplicity, but it is not able to reproduce ideal sensitivity and specificity. WINGS project results found DIPSI has very low sensitivity of $22.6 \%$ and specificity of $97.8 \%$ whereas IADPSG criteria has sensitivity of $27.7 \%$ and specificity of $97.7 \%$.

The lower sensitivity of DIPSI is believed to be due to non fasting state when women consumes a carbohydrate meal, insulin level rises due to increased blood glucose levels and when a glucose load of 75 grams given at this point, blood glucose levels are fluctuated since insulin levels are already elevated. The sensitivity of the test drops. Therefore, WINGS project supports the international guidelines where test should be done after over night fast.

Table 11: Prevalence of GDM in various studies.

\begin{tabular}{|llll|}
\hline Studies & No. of cases & DIPSI & IADPSG \\
\hline${\text { Geetha et } \mathrm{al}^{7}}^{7}$ & 100 & $14 \%$ & $9 \%$ \\
\hline Pulkit et $\mathrm{al}^{8}$ & 152 & $74.34 \%$ & $88.15 \%$ \\
\hline Present study & 144 & $17.4 \%$ & $15.3 \%$ \\
\hline
\end{tabular}

In another study of 1031 pregnant women from Chennai comparing DIPSI non fasting with IADPSG criteria for diagnosis of GDM found 10.3\% (106) women were diagnosed by IADPSG criteria. Out of them only $22.65 \%$ (24) women were diagnosed by DIPSI leaving behind $77.4 \%$ (82) women undiagnosed by DIPSI and they concluded that DIPSI has very low sensitivity compared to IADPSG criteria. ${ }^{5}$ Wide variation in GDM prevalence rates may be attributed to the use of different criteria for diagnosis, variation in geographical region and lifestyle with lack of physical activity.

\section{CONCLUSION}

DIPSI is practically simple, easier, non fasting single test procedure, suitable in low resource countries. Though IADPSG criteria, is uniform criteria for screening and diagnosis of GDM worldwide the sensitivity of IADPSG criteria was found to be slightly lesser when compared with DIPSI criteria in present study.

Funding: No funding sources

Conflict of interest: None declared

Ethical approval: The study was approved by the Institutional Ethics Committee

\section{REFERENCES}

1. Metzger BE. Organizing committee: Summary and recommendations of the third international workshop conference on gestational diabetes mellitus. Diabetes. 1991;40 S2:197-201.

2. International Diabetes Federation. IDF Diabetes Atlas, 6th edn. Brussels, Belgium: International Diabetes Federation, 2013. Available at http:11 www.idf.org/sites/ default/files/ EW-6E-Atlas-Full0.Pdf. Accessed on 25 July 2014.

3. Madsen JK, Haunsoe S, Helquit S, Hommel E, Malthe I, Pedersen NT, et al. Prevalence of hyperglycemia and undiagnosed diabetes mellitus in patients with acute myocardial infarction. Acta Med Scand. 1986;220:329-32.

4. Zargar AH, Sheikh MI, Bashir MI, Massodi SR, Laway BA, Wani AI, et al. Prevalence of gestational diabetes mellitus in kashmiri women from the Indian subcontinent. Diabetes Res Clin Pract. 2004;66:13945.

5. Mohan V, Mahalakshmi MM, Bhavadharini B, Maheshwari K, Kaliyarasi G, Anjana RM, et al. Comparison of screening for gestational diabetes mellitus by oral glucose tolerance tests done in the non - fasting (random) and fasting states. Acta Diabetol. 2014;51:1007-13.

6. Goldberg RJ, Hanley AJG, Yec, Ziman B, Sermer $\mathrm{M}$, Retnakaran $\mathrm{R}$ et al. Circadian variation in the response to the glucose challenge test in pregnancy. Diabetes Care. 2012;35:1578-84.

7. Geetha N, Sangeetha KG. Comparison of IADPSG and DIPSI criteria for diagnosis of gestational diabetes mellitus. IOSR JDMS. 2016;15(9):1-4.

8. Vij P, Jha S, Gupta SK, Aneja A, Mathur R, Waghdhare S, et al. Comparison of DIPSI and 
IADPSG criteria for diagnosis of GDM: A study in a north Indian tertiary center. Int $\mathrm{J}$ Diabetes Dev Ctries. 2015;35(3):285-8.

9. Polur H, Rrora R, Vinodh Bandela P. A mini review on diagnostic criteria of gestational diabetes mellitus. J Pharm Sci Res. 2015;7(8):538-41.

10. Bhavadharini B, Uma R, Saravanan P, Mohan V. Screening and diagnosis of gestational diabetes mellitus-relevance to low and middle income countries. Clini Diabetes Endocrinol. 2016;2(13):1-8.

Cite this article as: Srinivasan S, Reddi RP.

Comparative study of DIPSI and IADPSG criteria for diagnosis of GDM. Int J Reprod Contracept Obstet Gynecol 2018;7:932-7. 